Canadian

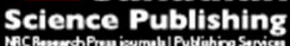

Applied Physiology, Nutrition, and Metabolism Physiologie appliquée, nutrition et métabolisme

\title{
Minimal Alteration in Muscle Lipid Genes Following Stabilized Weight Loss
}

\begin{tabular}{|c|c|}
\hline Journal: & Applied Physiology, Nutrition, and Metabolism \\
\hline Manuscript ID & apnm-2017-0098.R2 \\
\hline Manuscript Type: & Article \\
\hline Date Submitted by the Author: & 18-Jun-2017 \\
\hline Complete List of Authors: & $\begin{array}{l}\text { Coker, Robert; University of Alaska Fairbanks, Institute of Arctic Biology; } \\
\text { University of Arkansas for Medical Sciences, Geriatrics } \\
\text { Robinette, Leizleigh; University of Arkansas for Medical Sciences, Geriatrics } \\
\text { Kern, Philip; University of Kentucky College of Medicine, Internal Medicine }\end{array}$ \\
\hline \multicolumn{2}{|l|}{$\begin{array}{l}\text { Is the invited manuscript for } \\
\text { consideration in a Special } \\
\text { Issue? : }\end{array}$} \\
\hline Keyword: & $\begin{array}{l}\text { nutrition }<\text { nutrition, fat metabolism }<\text { metabolism, mitochondrial } \\
\text { metabolism }<\text { metabolism, obesity }<\text { waist circumference, metabolic } \\
\text { syndrome }\end{array}$ \\
\hline
\end{tabular}


Minimal Alteration in Muscle Lipid Genes Following Stabilized Weight Loss

Robert H. Coker ${ }^{1,2}$, Leizleigh Robinette ${ }^{2}$, and Philip A. Kern ${ }^{3}$

Institute of Arctic Biology ${ }^{1}$, University of Alaska-Fairbanks, Fairbanks, AK; Center for Translational Research in Aging and Longevity, Department of Geriatrics ${ }^{2}$, University of Arkansas for Medical Sciences, Little Rock, AR; Department of Internal Medicine, Division of Endocrinology ${ }^{3}$, University of Kentucky, Lexington, KY

Running Head: Physical Activity, Caloric Restriction and Metabolism

Manuscript Word Count: 3150

Abstract Count:

Address for Correspondence:

Robert H. Coker, Ph.D.

Institute of Arctic Biology

University of Alaska-Fairbanks

902 North Koyukuk Drive

email: rcoker@alaska.edu

Fairbanks, AK 99775-7000

Telephone \# 907-474-6701

FAX \# 907-474-5700 


\section{ABSTRACT}

Purpose: Variations in skeletal muscle peroxisome proliferator-activated receptor gamma coactivator 1-alpha $(\mathrm{PGC}-1 \propto)$, carntine palmitoyltransferase $(\mathrm{CPT}-1)$, perilipin protein $(\mathrm{PLIN} 2)$, and adipose tissue triglyceride lipase (ATGL), and comparative gene identification-58 (CGI-58) have been described to play important roles in the metabolic regulation of lipid oxidation, and may influence intramyocellular lipid (IMCL) and muscle lipid droplet size (LDS). While acute changes in caloric balance and/or aerobic capacity may affect lipid metabolism, the influence of sustained weight loss derived from caloric restriction (CWL) compared to exercise training (EWL) on the above mentioned parameters has not been fully elucidated.

Methods: Using a combination of metabolic feeding and/or supervised exercise training, we evaluated the influence of stabilized weight loss elicited by CWL compared to EWL without the confounding influence of acute alterations in caloric balance on molecular markers of mitochondrial metabolism and lipid droplet size in middle-aged overweight individuals with impaired glucose tolerance.

Results: There were no significant changes in PGC-1 $\propto$, CPT-1, PLIN2, ATGL and CGI-58 mRNA in CWL and EWL. While there were no changes in ATGL mRNA in CWL, there was a strong trend $(\mathrm{P}=0.05)$ for the $\triangle \mathrm{ATGL}$ mRNA in EWL with stabilized weight loss. There were no significant changes in IMCL or LDS within skeletal muscle in CWL or EWL, respectively. 
Conclusion: Under the conditions of chronic caloric balance following dietary or exercise-based interventions, mediators of mitochondrial function, IMCL and LDS were largely unaffected.

Future studies should focus on intervention-based changes in protein expression and/or phosphorylation, and the relationship to physiological endpoints.

Key words: Nutrition, fat metabolism, mitochondrial metabolism, obesity, metabolic syndrome 


\section{INTRODUCTION}

Concomitant changes in physical activity and dietary intake that result in a caloric deficit

will reduce adipose tissue and improve glucose tolerance in insulin resistant individuals (Hays et al. 2008). While exercise and dietary modification may be beneficial in the prevention or the mitigation of metabolic disease (Amati et al. 2011; He et al. 2004), the majority of individuals use caloric restriction alone in their attempt to lose weight (Weiss et al. 2006). This is unfortunate as exercise-induced enhancements in mitochondrial biogenesis and improvements in oxidative enzymes were demonstrated in pre-clinical models (Holloszy 1976), and these adaptations have now been linked to favorable improvements in lipid turnover within skeletal muscle in obese individuals (Listenberger et al. 2007).

Metabolic disease may be linked to mitochondrial dysfunction, and this relationship has been posited to represent a key element responsible for muscle atrophy and insulin resistance in obese individuals (Di Gregario et al. 2005; Lin 2002; Schenk and Horowitz 2007). Ceramide and diacylglycerol (DAG) may accumulate under these circumstances due to increased fatty acid delivery and/or inadequate fatty acid oxidation, activate protein kinase $\mathrm{C}$, and this sequalae been posited as a potential mechanism in the pathogenesis of insulin resistance (Hegarty et al. 2003;

Itani et al. 2002). Referred to as the "athletes paradox", studies have demonstrated reorganization of IMCL and DAG in lean individuals compared to obese sedentary individuals (Amati et al. 
2011; Devries et al. 2013). Moreover, 8 weeks of aerobic exercise training in obese individuals reduced IMCL content by $\sim 40 \%$, improved lipolytic protein expression, and points to the potential importance of increased lipid flux with regard to improvements in metabolic health (Louche et al. 2013). Despite these interesting data that may or may not include any significant weight loss, the influence of caloric restriction on muscle lipotoxicity in obese individuals at risk for the development of metabolic disease has received even less attention (Solomon et al. 2008).

The mediators at play within the etiology of muscle lipotoxicity are complex. Peroxisome proliferator-activated receptor coactivator (PGC-1 $\alpha)$ has been shown to promote increased mitochondrial biogenesis (Hood 2001). In fact, exercise training (acute and chronic) will increase PGC-1 $\alpha$ mRNA in skeletal muscle (Larsen et al. 2012; Pilegaard et al. 2003; Tunstall et al. 2002). Conversely, short-term bed rest results in a significant decrease in PGC-1 $\alpha$ mRNA (Ringholm et al. 2011). Carnitine palmitoyl transferase-1 (CPT-1) mRNA has been shown to increase substantially with exercise training along with significant improvements in total lipid oxidation (Holloszy 2008). With exercise training, PGC-1 $\alpha$-mediated improvements in mitochondrial biogenesis may work cohesively with increases in CPT-1 that is responsible for the transport of long chain acylcarnitine derived from long chain fatty acids into the mitochondria (Lass et al. 2011). Other important proteins include perilipin 2 (PLIN2) (Listenberger et al. 2007), which respond to exercise training and help shift FFA metabolism 
between storage and utilization (Ducharme et al. 2008). While the surface of lipid droplets may contain PLIN2 that limits the interaction with adipose tissue triglyceride lipase (ATGL) (Louche et al. 2013), preferential utilization of PLIN2-associated lipid droplets has been demonstrated during exercise in humans (Shaw et al. 2012). Instead of acute alterations in caloric balance through dietary or exercise training that have already been shown to increase mitochondrial function (Lass et al. 2011; Pilegaard et al. 2003; Tunstall et al. 2002), we hypothesized that stabilized weight loss would promote sustained improvements in muscle lipid genes. Moreover, we also hypothesized that stabilized weight loss following exercise training would initiate even greater increments in PGC-1 $\alpha$, CPT-1, PLIN2 and ATGL when compared to stabilized weight loss from diet alone. In turn, sustained improvements in mRNA would reduce lipotoxicity in skeletal muscle that could ultimately be relevant to overall improvements in metabolic health (Petersen et al. 2007). 


\title{
Materials and Methods
}

\author{
Research Volunteers
}

We recruited twenty men and women aged 50-80 yr who were overweight with a BMI range of $26-40 \mathrm{~kg} / \mathrm{m}^{2}$ who were weight stable over the last six months, nonsmokers, sedentary ( $\leq 2$ day/wk of structured physical activity) from the central Arkansas area through the use of newspaper advertisements. Participants were recruited without regard to sex, race, or ethnic status. Screening procedures required for participation included a medical history, physical examination, routine blood and urine chemistries, oral glucose tolerance test (OGTT), and a maximal exercise test on a bicycle ergometer. With regard to the OGTT, all individuals with a plasma glucose concentration of less than $200 \mathrm{mg} / \mathrm{dl}$ at $120 \mathrm{~min}$ after the consumption of oral glucose but who were otherwise healthy, were eligible for participation in the study. Participants with a history of cardiovascular disease, diabetes, or other significant medical problem, and anyone taking medicines or supplements that might have had potential effects on carbohydrate or lipid metabolism were excluded were excluded. Each subject provided written informed consent, and study procedures were approved by the Institutional Review Board of the University of Arkansas for Medical Sciences (UAMS) and the Central Arkansas Veterans Healthcare System Research and Development Committee 


\section{Experimental Protocol}

The experimental protocol and the data related to body composition and insulin

sensitivity has been published previously (Coker et al. 2009). Briefly, eligible participants were randomly assigned into one of three groups: 1) exercise training with weight loss (EWL);2) caloric restriction with weight loss (CWL) and controls. While no attempt was made to affect dietary intake or physical activity in the control group, we utilized a combination of metabolic feeding (CWL and EWL) and supervised exercise training (EWL) in the intervention groups.

Except for controls, a weight maintenance diet was followed for 4 weeks by all subjects before pre intervention testing. Body weights were recorded on a weekly basis, and adjustments to the weight maintenance diet were made to ensure caloric balance by the end of the 4 week period. In CWL, the reduction in caloric intake began immediately after the completion of the weight maintenance phase and the pretesting session. After the weight maintenance phase and pre intervention testing, subjects randomized to EWL began their exercise training regimen at $50 \%$ of peak oxygen consumption $\left(\mathrm{VO}_{2 \text { peak }}\right)$ on a bicycle ergometer. All individuals completed an assessment of $\mathrm{VO}_{2 \text { peak }}$ in order to supervise exercise training at a specific workload that could be used to determine caloric expenditure. In order to facilitate optimal compliance, we gradually increased kcal expenditure with exercise training (ie., first week at $1000 \mathrm{kcal} / \mathrm{week}$ and elevated by $500 \mathrm{kcal} /$ week until total energy expenditure reached $2500 \mathrm{kcal} /$ week). In CWL, we matched 
the progressive increase in energy expenditure in EWL with a progressive caloric deficit.

Therefore, the weekly and 12-week theoretical caloric deficit of the subjects in the CWL and EWL groups were identical. Following the 12-week weight loss period, participants in the CWL and EWL groups underwent a 2-wk of gradual refeeding phase followed by a 4-wk weight stabilization phase. These refeeding and weight stabilization phases allowed participants to reestablish a stable, yet significantly reduced body weight. This was necessary since an acute hypocaloric state has been shown to contribute to improved glycemic control (Hays et al. 2008). Thus, subjects in the EWL group actually exercised for 18 weeks (ie., 12 weeks under caloric deficit, 2 weeks of gradual adjustment to caloric balance and 4 weeks at caloric balance). Postintervention testing sessions for EWL subjects were completed approximately $72 \mathrm{~h}$ after the last exercise session under conditions of stabilized weight loss (Coker et al. 2009).

In the control group, caloric and macronutrient intake was standardized through consumption of a mixed diet (35\% fat, $20 \%$ protein, $45 \%$ carbohydrate) $4 \mathrm{~d}$ before pre- and post testing sessions. Controls were advised to maintain their normal diet during the rest of the study and non-exercising subjects were instructed to maintain their habitual physical activity. All other volunteers consumed a similar diet with respect to the macronutrient profiles and a weight maintenance diet for a 4-wk period before pre-intervention testing. For all of the dietary feeding in the study, the Nutritional Services component of the Clinical Research Services Core at 
UAMS provided a 3-d rotation diet based on the Harris-Benedict equation throughout the study. An activity factor (AF) of 1.6 during diet stabilization was used to estimate the total daily energy requirement according to the following equation: $=[66+(13.7 \mathrm{x}$ weight in $\mathrm{kg})+(5 \mathrm{x}$ height in $\mathrm{cm})-(608 \mathrm{x}$ age in $\mathrm{yr})] \mathrm{x}$ AF (Coker et al. 2009). All subjects were trained under direct supervision at the Donald W. Reynolds Institute on Aging using a cycle ergometer. There were no adverse events during the study.

Muscle biopsy samples were collected in the fasting condition at week 6 (preintervention) and week 24 (post-intervention) under local anesthetic (1\% Xylocaine $\mathrm{HCl}$ ) using a $6 \mathrm{~mm}$ needle as described by Evans et al. (Evans et al. 1986).

Analysis

We isolated the total RNA from muscle biopsies using an Ultraspec RNA Isolation System kit (Biotex, Houston, TX). The quantity and quality of the isolated RNA were determined by Agilent 2100 Bioanalyzer (Palo Alto, CA). Real-time RT-PCR and the primer sequences of 18S were performed as described previously (Borengasser et al. 2011). We generated standard curves by the utilization of pooled RNA from the samples assayed. In this way, the data generated represent arbitrary units that can be used to accurately compare each set of samples to each other. All data were expressed in relation to $18 \mathrm{~S}$ RNA, and all samples were analyzed 
twice with and without reverse transcriptase. No amplification was seen in the samples in the absence of reverse transcriptase.

Skeletal muscle biopsy samples were immediately processed for fiber typing and lipid content analysis, using Oil Red O staining and imaging techniques (Di Gregario et al. 2005; Kerner and Hoppel 2000). The values for IMCL were expressed as the total area of lipid droplets in a muscle fiber by the total area of the same muscle fiber. The oil red O staining intensity of either type 1 or 2 muscle fibers was quantified using National Institutes of Health Image program (http://rsb.info.nih.gov/nih-image/). We utilized confocal laser scanning microscopy (540 to $580 \mathrm{~nm}$ ) and a quantitative image analysis program (Northern Eclipse 6.0) to determine the size of lipid droplets as previously described by (He et al. 2004).

Data related to glucose metabolism and respiratory quotients from the $\mathrm{VO}_{2}$ peak during the graded exercise test were extracted from (Coker et al. 2009) have been provided to match the participants in the current study (Table 1).

Statistical comparisons regarding mRNA, IMCL, lipid droplet size, weight, BMI, $\mathrm{VO}_{2 \text { peak, }}$ and insulin-stimulated glucose disposal (ISGD) between groups and over time were made using two-way analysis of variance. Bonferroni post hoc tests were applied to significant group x time interactions. Data are reported as means \pm SEM. Statistical significance was established at $P=0.05$. 


\section{Results}

Human Participants. Muscle tissue was available from 18 of the 20 total participants who were originally enrolled for the study (13 females and 5 males, mean age $57 \pm 1 \mathrm{yr}$, and mean BMI

$31 \pm 1 \mathrm{~kg} / \mathrm{m}^{2}$ ) (Table 1). Two volunteers were unable to maintain adequate compliance (ie., $80 \%$ compliance to feeding and/or training sessions) to the protocol. Participants were followed closely and compliance with the protocol was excellent. As previously published, volunteers randomized to CWL and EWL lost an equivalent amount of body weight and remained weight stable for 4 weeks prior to post-intervention. This strategy eliminated the influence of acute caloric restriction on metabolism and allowed us to delineate the influence of weight loss achieved through caloric restriction versus exercise training (Coker et al. 2009).

Molecular Regulation. PGC-1œ, CPT-1, PLIN2, CGI-58 and ATGL mRNA. There were no differences $(P>0.05)$ in PGC-1 $\propto$, CPT-1, and PLIN2 (Figure 1). Also, there were no differences in CGI-58 and ATGL between groups or in response to the CWL or EWL interventions (Figure 2). There was a trend toward significance (ie., $P=0.053$ ) when a paired t-test was used to evaluate changes in ATGL from baseline in EWL.

IMCL and Lipid droplet size. There were no significant differences $(P>0.05)$ in the \% IMCL in Type 1 or Type 2 muscle fibers between the control, CWL, or EWL groups from pre- to postintervention (Figure 3). There was a significant difference (ie., $P=0.03$ ) in the baseline values for 
the \% IMCL in Type 1 muscle fibers between control and EWL (Figure 1). In terms of the absolute amount and change in the lipid droplet size from pre- to post-intervention, there were no significant differences (Figure 4).

\section{Discussion}

Numerous studies have demonstrated the powerful influence of exercise training and/or caloric deficit on the regulation of the indices of mitochondrial metabolism (Russell et al. 2014). We have also shown significant improvements in $\mathrm{VO}_{2 \max }$ that were linked to enhancements in hepatic and peripheral insulin sensitivity (Coker et al. 2009). Nonetheless, it has been difficult to ascertain whether these changes in mitochondrial metabolism persist following the intervention under conditions of chronic caloric balance. In the present study, we hypothesized that PGC-1œ, CPT-1, PLIN2, CGI-58 and ATGL mRNA would be greater with exercise training compared to caloric restriction following identical weight loss interventions under weight stable conditions.

While our results would be much more definitive with the addition of data relative to changes in actual protein content, we found no changes in PGC-1 $\propto$, CPT-1, PLIN2, CGI-58 mRNA and no differences in IMCL or lipid droplet size between controls, CWL or EWL.

Previous studies have identified increases in mitochondrial genes, including PGC1 $\alpha$, in muscle following exercise, however most previous studies have examined acute exercise. In this study, we did not observe changes in most lipid genes, however we studied the effects of 
sustained exercise or weight loss. While significant improvements in mitochondrial content have been well characterized (Perry et al. 2010) and largely associated with the additive influence of acute bouts of aerobic exercise on mitochondrial biogenesis (Amati et al. 2011), it is likely that these effects are transient (Perry et al. 2010; Pilegaard et al. 2003) and therefore not detectable after individuals return to conditions of chronic caloric balance.

The powerful, yet transient exercise-induced increases in PGC- $1 \propto$ transcription and mRNA in human skeletal muscle has been previously identified (Pilegaard et al. 2003). It has also long been recognized that increased and sustained levels of physical activity have a profound influence on the overall regulation of mitochondrial metabolism in skeletal muscle (Holloszy 2008), but the temporary nature of these responses and how they relate to physiological functions under conditions of caloric balance may not be fully appreciated. Given that exercise-induced increments in CPT-1 activity were demonstrated over ten years ago (Bezaire et al. 2004), we anticipated elevations in CPT-1 mRNA to persist following our chronic exercise intervention. Unfortunately, the lack of data relative to actual protein content significantly limits our conclusions based on mRNA data. It could be that the exercise-induced influence on CPT-1 was post-translational or that middle-aged individuals respond in accordance with their level of deconditioning (Schild et al. 2015). 
We found that PLIN2 mRNA did not increase with EWL or CWL. This was different than previous studies that suggested a favorable response in PLIN2 expression with exercise training (Shepherd et al. 2012; Solomon et al. 2008). However, there are some important differences between the exercise interventions per se. For example, we utilized an exercise intensity of 50\% $\mathrm{VO}_{2 \text { peak }}$ in our studies (Coker et al. 2009) whereas other studies have utilized more vigorous exercise (Shepherd et al. 2013). Although we did not find significant changes in mRNA levels following stabilized weight loss in CWL and EWL, post-transcriptional changes could have promoted alterations in protein levels or enzyme activity during conditions in which caloric balance was negative (Hood et al. 2001).

Recognizing that the impact of CWL and EWL on insulin sensitivity was equally beneficial with respect to improvements in insulin sensitivity (Coker et al. 2009), and that previous studies have demonstrated concomitant enhancements in hormone sensitive lipase (HSL) and ATGL expression (Louche et al. 2013), it is interesting that ATGL mRNA was only slightly elevated by EWL. The sequestration of lipid in droplets provides a source of stored energy that is largely regulated based on metabolic need. Therefore, the weight stabilization period following CWL may have influenced the response such that the message for responsible for the regulation of intracellular triglyceride metabolism may be reset. 
There were no significant changes in IMCL or lipid droplet size in either group and no differences between the two groups. Other studies utilizing exercise and dietary interventions that elicited significant weight loss, and improvements in $\mathrm{VO}_{2}$ max , lipids and metabolic flexibility have also reported no change in IMCL (Solomon et al. 2008). When combined with exercise, short term alterations in dietary intake that utilize foods with a low glycemic index promoted an increase in IMCL (Haus et al. 2011). Remarkably, these studies also described a decrease in extramyocellular lipid (EMCL) and contributed to an increase in the IMCL/EMCL ratio. This may suggest lipid redistribution due to intervention that could be more predictive of beneficial changes in lipid metabolism and insulin sensitivity. Unfortunately, we did not measure EMCL in the present study. Also, we utilized Oil Red O staining instead of magnetic resonance spectroscopy and the differences in methodology should be recognized.

In previous studies, perilipin 2 (PLIN2) overexpression promoted an increase in IMCL and insulin sensitivity (Shepherd et al. 2012), and these results were potentially similar to results from studies utilizing acute exercise that promote triglyceride synthesis in skeletal muscle with simultaneous reductions in diacylglycerol and ceramide (Schenk and Horowitz 2007). It is interesting that despite significant improvements in hepatic and peripheral insulin sensitivity, the amount of IMCL and LDS was not affected. This is consistent with previous findings that 
indicate minimal alterations in lipid metabolism within skeletal muscle despite rapid systemic insulin resistance induced by overeating (Cornford et al. 2013).

We recruited a cohort of participants with a significant degree of insulin resistance. We anticipated a significant opportunity for reasonable improvements in molecular indices of mitochondrial metabolism as described by variations in mRNA, IMCL and lipid droplet size. In the scenario of chronic caloric balance following either weight loss intervention (caloric restriction or exercise training), this was not the case. While changes in mRNA may be helpful in the understanding of the molecular regulation of physiological processes, degradation of an exercise-induced alteration in CPT-1 mRNA may have already occurred at the time of data collection in this study.

In the current study, it would have been informative to evaluate post-intervention changes in mRNA immediately after the exercise intervention. Unfortunately, we did not perform a muscle biopsy at this time. As a result, we cannot distinguish acute (altered caloric balance) from chronic (caloric balance) differences in intervention (EWL vs CWL) - induced differences in PGC-1 $\propto$, CPT-1, PLIN2, and CGI-58 mRNA or IMCL and lipid droplet size. Future studies that employ exercise and caloric restriction are needed to determine whether changes in mRNA may only be associated with acute responses to these interventions, and how long changes in actual protein expression may affect physiological function. 
1. ACKNOWLEDGEMENTS: Funding was provided by National Institutes of Health, National Institute of Diabetes, Digestive and Kidney Diseases K01 DK 64716-01, NIH grants P30 AG28718 and UL1TR001998, and American Heart Association Grant 0335172N. We would like to thank Rick Williams, Bradley Hogate and Bounleut Phanavanh for expert technical analysis.

2. CONFLICT OF INTEREST: Dr. Coker is a Managing Partner and Co-Owner of Essential Blends, LLC that has received funding from the National Institutes of Health Small Business Innovations in Research program to develop clinical nutrition products. The data presented in this manuscript are unrelated. We declare that the results of the study are presented clearly, honestly, and without fabrication, falsification, or inappropriate data manipulation. 
References

Amati, F., Dubé, J.J., Alvarez-Carnero, E., Edreira, M.M., Chomentowski, P., Switzer, GE., et

al. 2011. Skeletal muscle triglycerides, diacylglycerol, and ceramides in insulin resistance,

another paradox in endurance trained athletes? Diabetes, 60: 10. 2588-2597.

doi: 10.2337/db11221. PMID 21873552.

Bezaire, V., Heigenhauser, G., Spriet, L., 2004. Regulation of CPT-I activity in intermyofibrillar and sarcolemmal mitochondria from human skeletal muscle. Am. J. Physiol. Endocrinol. Metab.

286: E85-E91. doi: 10.1152ajpendo.00237.2003. PMID: 12954596.

Borengasser, A., Varma, V., Coker, RH., Ranganathan, G., Phanavanh, B., Rasouli, N., et al.

2011. Adipose tissue triglyceride lipase expression in human adipose tissue and muscle. Role in insulin resistance and response to training and pioglitazone. Metabolism, 60: 1012-1020. doi:

10.1016/j.metabol.2010.10.005. PMID: 21129760.

Coker, RH., Williams, RH., Yeo, S., Kortebein, PM., Bodenner, DL., Kern. PA., et al.

2009. The impact of exercise training compared to caloric restriction on hepatic and peripheral insulin resistance in obesity. J. Clin. Endocrinol. Metab. 94: 4258-4266. doi: 10.1210/jc.20082033. PMID: 19808853.

Cornford, AS., Hinko, A., Nelson, RK., Barkan, AL., Horowitz, JF. 2013 Rapid development of systemic insulin resistance with overeating is not accompanied by robust changes in skeletal 
muscle glucose and lipid metabolism. Appl. Physiol. Nutr. Metab. 38: 512-519. doi:

10.1139/apnm-2012-0266. PMID: 23668758.

Devries, MC., Samjoo, IA., Hamadeh, MJ., McCready, C., Raha, S., Watt, MJ., et al. 2013.

Endurance training modulates intramyocellular lipid compartmentalization and morphology in

skeletal muscle of lean and obese women. J. Clin. Endocrinol. Metab. 98: 12. 4852-4862.

doi: 10.1210jc.2013-2044. PMID: 24081737.

Di Gregario, GB., Yao-Borengasser, A., Rasouli, N., Varma, V., Lu, T., Miles, LM., et al. 2005.

Expression of CD68 and macrophage chemoattractant protein-1 genes in human adipose and muscle tissues: association with cytokine expression, insulin resistance, and reduction by pioglitazone. Diabetes, 54: 2305-2313. PMID: 16046295.

Ducharme, N., Bickel, PE. 2008. Mini-Review: Lipid droplets in lipogenesis and lipolysis.

Endocrinology, 149: 942-949. doi: 10.1210/en.2007-1713. PMID: 18202123.

Evans, WJ., Meredith, CN., Cannon, JG., Dinarello, CA., Frontera, WR., Hughes, VA., et al.

1986. Metabolic changes following eccentric exercise in trained and untrained men. J. Appl.

Physiol. 61: 1864-1868. PMID: 3491061.

Haus, JM., Solomon, TP., Lu, L., Jesberger JA., Barkouskis H., Flask, CA., et al. 2011.

Intramyocellular lipid content and insulin sensitivity are increased following a short-term lowglycemic index diet and exercise intervention. Am. J. Physiol. Endocrinol. Metab. 301: E511- 
E516. doi: 10.1152/ajpendo.00221.2011. PMID: 21712533.

Hays, NP., Galassetti, PR., Coker, RH. 2008. Prevention and treatment of type 2 diabetes:

current role of lifestyle and pharmacological and natural product interventions. Pharmacol.

Ther. 118: 2. 181-191. doi: 10.1016/j.pharmthera.2008.02.003. PMID: 18423879.

He, J., Goodpaster, BH., Kelley, DE. 2004. Effects of weight loss and physical activity on

muscle lipid content and droplet size, Obes. Res. 12: 5. 761-769.

doi:10.1038/oby.2004.92. PMID: 15166296.

Hegarty, BD., Furler, SM., Ye, J., Cooney, GJ., Kraegen, EW. 2003. The role of intramuscular

lipid in insulin resistance. Acta. Physiol. Scand. 178: 4. 373-383. doi: 10.1046/j.1365-

201X.2003.01162.x.

Holloszy, J. 1976. Biochemical adaptations in muscle: effects of exercise on mitochondrial oxygen uptake and respiratory enzyme activity in skeletal muscle. J. Biol. Chem.

242: 2278-2282. PMID: 4290225.

Holloszy, J. 2008. Regulation by exercise of skeletal muscle content of mitochondria and

GLUT4. J. Physiol. Pharmacol. 59: 5-18. PMID: 19258564.

Hood, DA. 2001. Invited Review: contractile activity-induced mitochondrial biogenesis in skeletal muscle. J. Appl. Physiol. 90: 1137-1157. PMID: 11181630. 
Itani, SI., Ruderman, NB., Schmieder, F., Boden, G. 2002. Lipid-induced insulin resistance in human muscle is associated with changes in diacylglycerol, protein kinase $\mathrm{C}$, and IkappaB-alpha.

Diabetes, 51: 7. 2005-2011. PMID: 12086926.

Kern, PA., Simsolo, RB., Fournier, M. 1999. Effect of weight loss on muscle fiber type, fiber size, capillarity, and succinate dehydrogenase activity in humans. J. Clin. Endocrinol. Metab. 84: 11. 4185-4190. doi: 10.1210/jcem.84.11.6090. PMID: 10566670.

Larsen S, Nielsen J, Hansen, CN., Nielsen, LB., Wibrand, F., Stride, N., et al. 2012. Biomarkers of mitochondrial content in skeletal muscle of healthy young human subjects. J. Physiol.

590: 14, 3349-3360. doi: 10.1113/jphysiol.2012/230185. PMID: 22586215.

Lass, A., Zimmerman, R., Oberer, M., Zechner, R. 2011. Lipolysis - a highly regulated multienzymecomplex mediates the catabolisms of cellular fat stores. Prog. Lipid Res. 50: 4-27.

Lin, J., Wu, H., Tarr, PT., Zhang, CY, Wu, Z., Boss, O., et al. 2002. Transcriptional co-activator PGC-1 drives the formation of slow-twitch muscle fibres. Nature, 418: 797-801. doi:

10.1038/nature00904. PMID: 12181572. 
Listenberger, LL., Ostermeyer-Fay, AG., Goldberg EB., Brown, WJ., Brown, DA. 2007.

Adipocyte differentiation-related protein reduces the lipid droplet association of adipose

triglyceride lipase and slows triacylglycerol turnover. J. Lipid Res. 48: 12. 2751-2761. doi:

10.1194/jlr.M700359-JLR200. PMID: 17872589.

Louche, K., Badin, PM., Montastier, E, Laurens, C., Bourlier, V., deGlisezinski, I., et al. 2013.

Endurance exercise training up-regulates lipolytic proteins and reduces triglyceride content in

skeletal muscle of obese subjects. J. Clin. Endocrinol. Metab. 98: 12. 4863-4871. doi:

10.1210/jc.2013-2058. PMID: 24178794.

Perry, CG., Lally, J., Holloway, GP., Heigenhauser, GJ., Bonen, A., Spriet, LL. 2010. Repeated transient mRNA bursts precede increases in transcriptional and mitochondrial proteins during training in human skeletal muscle. J. Physiol. 588: (Pt 23). 4795-4710.

doi: 10.1113/jphysiol.2010.199448. PMID: 20921196.

Petersen, KF., Dufour, S., Savage, DB., Bilz, S., Solomon, G., Yonemitsu, S., et al. 2007. The role of skeletal muscle insulin resistance in the pathogenesis of metabolic syndrome. Proc. Natl.

Acad. Sci. USA. 104: 31. 12587-12594. doi: 10.1073/pnas.0705408104. PMID: 17640906.

Pilegaard, H., Saltin, B., Neufer, D. 2003. Exercise induces transient transcriptional activation of the PGC1-alpha gene in human skeletal muscle. J. Physiol. 546: (Pt 3). 851-858.

PMID: 12563009. 
Ringholm, S., Bienson, RS., Krilerich, K., Guadalupe-Grau, A., Aachmann-Andersen, NJ.,

Saltin, B., et al. 2011. Bed rest reduces metabolic protein content and abolishes exercise-induced

2 mRNA responses in human skeletal muscle. Am. J. Physiol. Endocrinol. Metab. 30: E649-

E658. doi: 10.1152/ajpendo.00230.2011. PMID: 21750272.

Russell, A., Foletta, RJ., Snow, RJ., Wadley, GD. 2014. Skeletal muscle mitochondria: A major player in exercise, health and disease. Biochim. Biophys. Acta. 1840: 4. 1276-1284. doi:

10.1016//bbagen.2013.11.016. PMID: 24291686.

Schenk, S., Horowitz, JF. 2007. Acute exercise increases triglyceride synthesis in skeletal muscle and prevents fatty acid-induced insulin resistance. J. Clin. Invest. 117: 6. 1690-1698. doi: 10.1172/JCI30566. PMID: 17510709.

Schild, M., Ruhs, A., Beiter, T., Zügel, M., Hudemann, J., Reimer, A., et al. 2015. Basal and exercise induced label-free quantitative protein profiling of $\mathrm{m}$. vastus lateralis in trained and untrained individuals. J. Proteomics, 122: 119-132. doi: 10.1016/j.prot.2015.03.028.

PMID: 25857276.

Shaw, CS., Shepherd, SO., Wagenmakers, AJ., Hansen, D., Dendale, P., van Loon, LJ. 2012.

Prolonged exercise training increases intramuscular lipid content and perilipin 2 expression in type 1 muscle fibers of patients with type 2 diabetes. Am. J. Physiol. Endocrinol. Metab. 303:

9. E1158-E1165. doi: 10.1152/ajpendo.00272.2012. PMID: 22949030. 
Shepherd, SO., Cocks, M., Tipton, KD., Ranasinghe, AM., Barker, TA., Burniston, JG., et al. 2012. Preferential utilization of perilipin2-associated intramuscular triglycerides during 1-h of moderate-intensity endurance-type exercise. Exp. Physiol. 97: 8. 970-982. doi:

10.1113/expphysiol.2012.064592. PMID: 22496505.

Shepherd, SO., Cocks, M., Tipton, KD., Ranasinghe, AM., Barker, TA., Burniston, JG., et al. 2013. Sprint interval training and traditional endurance training increase net intramuscular riglyceride breakdown and expression of perilipin 2 and 5. J. Physiol. 591: 3. 657-675. doi: 10.1113/jphysiol.2012.240952. PMID: 23129790.

Solomon, TP., Sistrun, SN., Krishnan, RK., Del Aguila, LF., Marchetti CM., O'Carroll SM., et al. 2008. Exercise and diet enhance fat oxidation and reduce insulin resistance in older obese adults. J. Appl. Physiol. 104: 5. 1313-1319. doi: 10.1152/japplphysiol.00890.2007.

PMID: 18323464.

Solomon, TP., Haus, JM., Cook, MA., Flask, CA., Kirwan, JP. 2013. A low-glycemic diet lifestyle intervention improves fat utilization during exercise in older obese humans. Obesity (Silver Spring). 21: 11. 2272-2278. doi: 10.1002/oby.20411. PMID: 23512711.

Tunstall, RJ., Mehan, KA., Wadley, GD., Collier, GR., Bonen, A., Hargreaves, M., et al. 2002. Exercise training increases lipid metabolism gene expression in human skeletal muscle. Am. J.

Physiol. Endocrinol. Metab. 283: 1. E66-E72. doi: 10.1152/ajpendo.00475.2001. 
PMID: 12067844.

Weiss, EC., Galuska, DA., Khan, LK., Serdula, MK. 2006. Weight control practices among

US adults, 2001-2002. Am. J. Prev. Med. 31: 1. 18-24. doi: 10.1016/jamepre.2006.03.016.

PMID: 16777538. 
Table 1. Clinical Characteristics

\begin{tabular}{|c|c|c|c|c|c|c|}
\hline & $\begin{array}{l}\text { Con } \\
\text { Pre }\end{array}$ & Post & $\begin{array}{c}\text { EWL } \\
\text { Pre }\end{array}$ & Post & $\begin{array}{l}\text { CR } \\
\text { Pre }\end{array}$ & Post \\
\hline Gender (f/m) & $4 / 2$ & & $5 / 1$ & & $4 / 2$ & \\
\hline Race (C/AA) & $6 / 0$ & & $3 / 2$ & & $5 / 1$ & \\
\hline Age (yr) & $60 \pm 1$ & & $53 \pm 2$ & & $59 \pm 1$ & \\
\hline Weight (kg) & $86 \pm 5$ & $88 \pm 5$ & $85 \pm 6$ & $79 \pm 6^{*}$ & $86 \pm 2$ & $80 \pm 3^{*}$ \\
\hline BMI $\left(\mathrm{kg} / \mathrm{m}^{2}\right)$ & $30 \pm 1$ & $30 \pm 1$ & $33 \pm 2$ & $30 \pm 2^{*}$ & $30 \pm 1$ & $28 \pm 1^{*}$ \\
\hline $\mathrm{VO}_{2}(\mathrm{I} / \mathrm{min})$ & $1.9 \pm 0.2$ & $1.8 \pm 0.1$ & $1.6 \pm 0.1$ & $2.0 \pm 0.2^{*}$ & $1.8 \pm 0.2$ & $1.7 \pm 0.2$ \\
\hline $\mathrm{RQ}$ at $\mathrm{VO}_{2 \text { peak }}$ & $1.1 \pm 0.0$ & $1.1 \pm 0.0$ & $1.2 \pm 0.0$ & $1.2 \pm 0.0$ & $1.2 \pm 0.0$ & $1.2 \pm 0.0$ \\
\hline ISGD & $5.5 \pm 0.9$ & $4.9 \pm 1.2$ & $7.9 \pm 0.7$ & $10.2 \pm 0.9^{*}$ & $6.2 \pm 0.6$ & $7.3 \pm 0.6$ \\
\hline
\end{tabular}




\section{FIGURE LEGENDS}

Figure 1. PGC-1 alpha, CPT-1 and PLIN2 mRNA levels under weight stable, pre- and postintervention conditions in Control, EWL and CWL Value are expressed as mean $\pm \mathrm{SEM}$.

Figure 2. CGI-58 and ATGL levels under weight stable, pre- and post-intervention conditions in Control, EWL and CWL Value are expressed as mean \pm SEM.

Figure 3. Percent lipid in type 1 and type 2 fibers of the vastus lateralis under weight stable, preand post-intervention conditions in Control, EWL and CWL. *Represents a significant difference between pre-intervention values for control and EWL. Value are expressed as mean $\pm \mathrm{SEM}$.

Figure 4. Lipid droplet size in type 1 and type 2 fibers of the vastus lateralis under weight stable, pre- and post-intervention conditions in Control, EWL and CWL Value are expressed as mean \pm SEM. 

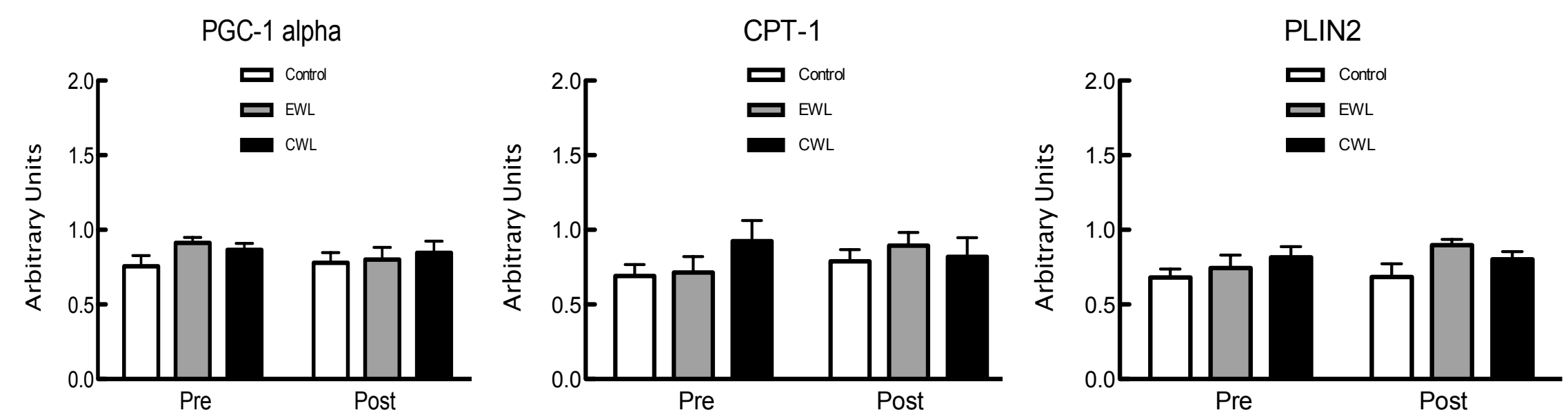

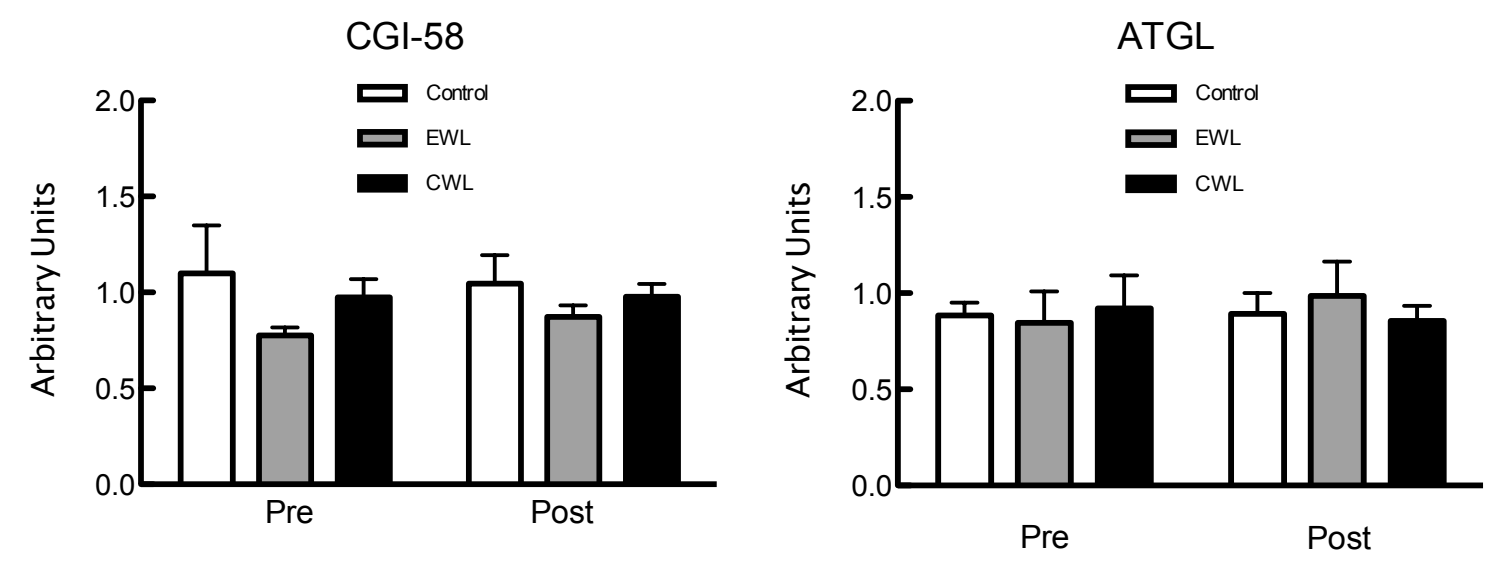


\section{Percent Lipid}

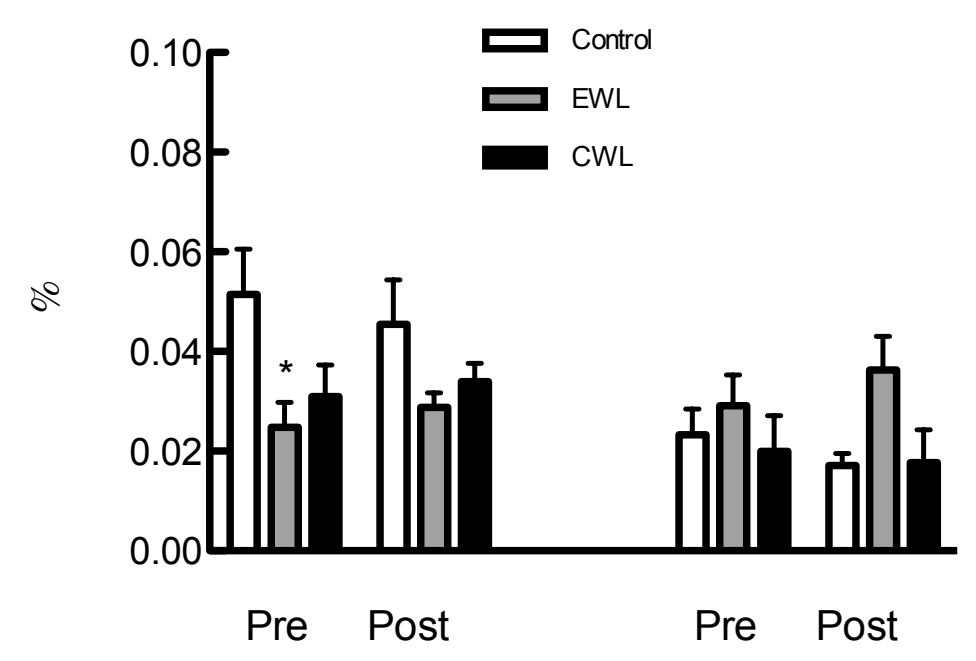

Type 1

Type 2 


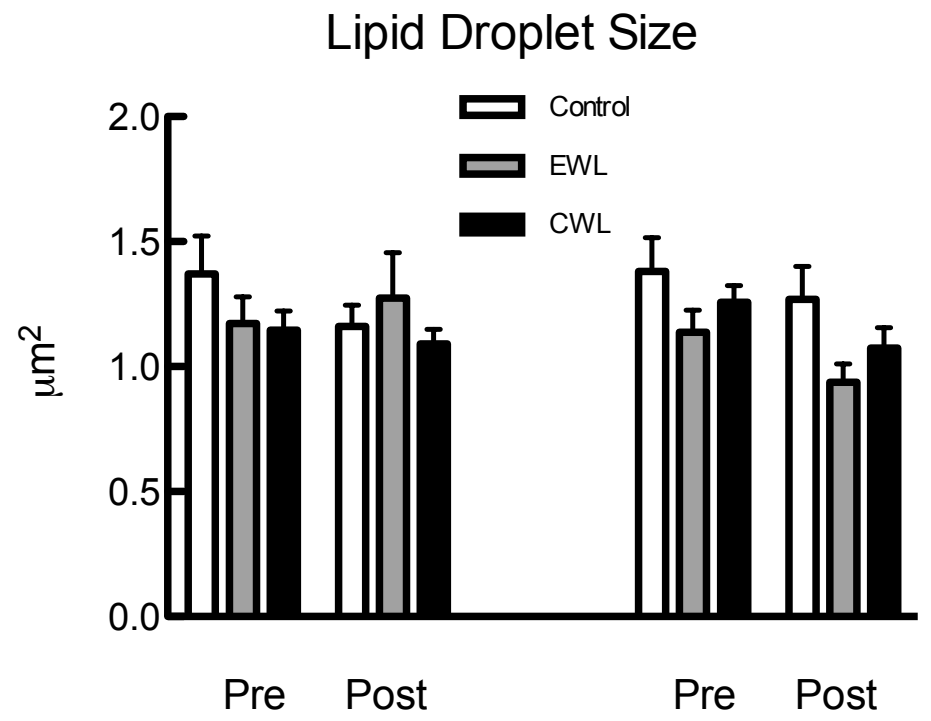

Type 1

Type 2 